\title{
Avaliação do grau de conhecimento que pacientes com diabetes mellitus demonstram diante das alterações oculares decorrentes dessa doença
}

\author{
Evaluation of the knowledge that patients with diabetes mellitus \\ demonstrate about ocular changes due to this illness
}

\author{
Gabriela Assumpção Brito Pereira ${ }^{1}$ \\ Renata Louise Battisti Archer ${ }^{2}$ \\ Cristiane Andrade Coelho Ruiz ${ }^{3}$
}

\begin{tabular}{l} 
RESUMO \\
\hline Objetivo: Avaliar o nível de informação que os pacientes portadores de \\
diabetes mellitus possuem em relação às complicações oculares oca- \\
sionadas por essa condição. Métodos: Foram avaliados 100 pacientes \\
diabéticos em três semanas, independente do tipo da doença, com con- \\
sulta previamente marcada e que compareceram para avaliação no Am- \\
bulatório de Endocrinologia do Hospital Guilherme Álvaro. O paciente \\
estando de acordo, foi iniciado um questionário com oito perguntas \\
voltadas ao seu conhecimento sobre as complicações oftalmológicas \\
decorrentes do diabetes mellitus. Resultados: Foram avaliados 62 \\
mulheres e 38 homens, $93 \%$ eram diabéticos tipo II, com média de idade \\
de 58,64 anos ( $\pm 15,64$ anos). Quarenta pacientes apresentavam a doença \\
há mais de 10 anos. Setenta pacientes, que conheciam a existência dessas \\
complicações, mencionaram principalmente a alteração retiniana e a \\
catarata secundária (respectivamente $30 \%$ e $27 \%$ ). Em relação às pergun- \\
tas sobre a possibilidade de tratamento, 30 pacientes relataram não \\
haver, 7 não sabiam opinar sobre o assunto e 63 responderam que as \\
alterações oculares podiam ser tratadas. Sobre as opções de terapêu- \\
tica possíveis, a cirurgia foi a mais citada (33\%), em seguida foram o uso \\
de óculos (26,6\%), aplicação de laser(19,4\%), uso de colírio(18\%) e lente \\
de contato (3\%). A maioria dos pacientes (56\%) associou a aquisição \\
dessas informações à orientação médica. Cinquenta e seis pacientes en- \\
tenderam a necessidade do acompanhamento especializado de 6 meses \\
a 1 ano e a média do tempo decorrido da última consulta foi de cerca de \\
1 ano e 1 mês ( \pm 1 ano). Setenta e cinco pacientes alegaram que o tempo \\
de doença apresentou analogia com o aparecimento das complicações \\
oculares. Quando interrogados sobre a realização de tratamentos oftal- \\
mológicos prévios, 78 entrevistados negaram e 12 asseguraram já terem \\
necessitado. Conclusões: Grande parte dos pacientes reconhece que \\
podem vir a ter complicações oculares pelo tempo prolongado de diabe- \\
tes mellitus ( $81 \%$ dos pacientes), mas há equivoco quantoà especificação \\
dessas e seus tratamentos.
\end{tabular}

Descritores: Diabetes mellitus/complicações; Retinopatia diabética, Catarata/epidemiologia; Conhecimentos, atitudes e prática em saúde

\section{INTRODUÇÃO}

Diabetes mellitus (DM) é um distúrbio crônico que se caracteriza pela modificação do metabolismo da glicose e de outras substâncias produtoras 
de energia, acarretando, a longo prazo, várias mudanças, especialmente vasculares e neurológicas ${ }^{(1)}$. Está associado ao comprometimento da produtividade, da qualidade de vida e da sobrevida dos indivíduos acometidos, além de envolver altos custos no seu tratamento e no das suas complicações que, em sua maior parte, são evitáveis ${ }^{(2)}$.

As mudanças na fisiologia ocular decorrentes do diabetes são devidas principalmente à opacificação do cristalino e às alterações vasculares retinianas; ambas podendo levar à baixa visão, reversível ou não. É considerado um importante problema de saúde pública, uma vez que sua taxa estimada prevalência, ajustada por idade, na população de 30 a 69 anos, segundo o Ministério da Saúde, é de 7,6 em 100 habi$\operatorname{tantes}^{(3)}$. Dessa forma, medidas de prevenção são necessárias e se mostram eficazes em reduzir o impacto desfavorável sobre a morbimortalidade destes pacientes ${ }^{(2)}$.

O DM constitui a principal causa de cegueira em indivíduos de 20 a 74 anos de idade nos Estados Unidos e, no Brasil, é a primeira causa de cegueira adquirida. As alterações retinianas são denominadas retinopatia diabética e atingem mais de $75 \%$ dos diabéticos, independente do tipo, com mais de 20 anos de doença ${ }^{(4)}$.

No ano de 2004, a Campanha da Federação Internacional de Diabetes no Dia Mundial do Diabetes (14 de novembro) enfocou as complicações oculares decorrentes dessa doença. Paralelamente, houve um programa para profissionais da rede básica de saúde do SUS, nos Municípios com população acima de 100 mil habitantes, visando capacitá-los para prevenção, diagnóstico, tratamento e controle desses agravos ${ }^{(5)}$.

Em relação à educação dos diabéticos sobre medidas preventivas e periodicidade do exame oftalmológico, o estudo publicado em junho de 2007 pelo Departamento de Oftalmologia da Universidade de São Paulo, envolvendo médicos e pacientes, mostrou que diabéticos tipo II apresentam nível de conhecimento satisfatório e os tipo I necessitam de maior orientação $^{(6)}$.

De acordo com Kanski, todos os diabéticos acima de 12 anos de doença ou com sintomas de perda visual devem ser avaliados por um oftalmologista. A frequência dos retornos vai depender do grau em que a doença se encontra ${ }^{(7)}$. É importante ressaltar que o sucesso do tratamento depende também da detecção precoce das lesões e do controle dos principais fatores de risco, como duração do diabetes, controle metabólico ineficaz, hipertensão arterial, tabagismo, obesidade e hiperlipidemia.

O diagnóstico e consequente condução da doença precoces só são possíveis se o paciente estiver ciente da necessidade do acompanhamento oftalmológico periódico

\section{OBJETIVO}

Avaliar o nível de informação que os pacientes portadores de diabetes mellitus possuem em relação às complicações oculares ocasionadas por essa condição.

\section{MÉTODOS}

O estudo prospectivo foi realizado no Ambulatório de Endocrinologia do Hospital Guilherme Álvaro, da Secretaria de Saúde do Estado de São Paulo, que funciona de segunda a sexta-feira, atendendo cerca de 20 pacientes por dia, entre primeira consulta e retorno.

Os critérios de inclusão foram pacientes diabéticos, independente do tipo da doença, com consulta previamente marcada e que compareceram para avaliação no ambulatório mencionado no dia da entrevista. Ao final, selecionamos e entrevistamos os 100 primeiros pacientes agendados, em um prazo de três semanas.

Antes do atendimento, eram convidados a participar de um trabalho de pesquisa, que consistia na resposta de um questionário composto por oito questões relacionado ao envolvimento ocular pelo diabetes. Uma só pessoa se responsabilizou pela entrevista.

Com o paciente de acordo, tendo assinado a autorização por escrito, o pesquisador iniciou as perguntas conforme o modelo anexo. As questões eram lidas aos entrevistados e as opções eram a eles propostas, restando então aos mesmos a escolha de uma ou mais alternativas (exceto nas questões 1,2,7 e 8, as quais eram conflitantes). Naquelas questões explicativas, a informação oferecida pelo paciente era anotada pelo pesquisador na área destinada.

\begin{tabular}{|c|}
\hline Questionário \\
\hline Nome (Iniciais): \\
\hline Idade:__anos Sexo: ( ) Masc ( ) Fem DM Tipo: ( )I ( ) II \\
\hline $\begin{array}{l}1 \text { - Quanto tempo sabe que tem diabete? } \\
\begin{array}{ll}\text { ( ) Até } 1 \text { ano } & \text { ( ) } 2 \text { a } 5 \text { anos } \\
\text { ( ) } 6 \text { a } 10 \text { anos } & \text { ( ) Mais de } 10 \text { anos }\end{array}\end{array}$ \\
\hline $\begin{array}{l}2 \text { - Você acha que o diabetes pode causar alterações nos olhos? } \\
\begin{array}{ll}\text { ( ) Sim ( ) Não } & \text { ( ) }\end{array}\end{array}$ \\
\hline $\begin{array}{l}3 \text { - Se sim, quais tipos de alterações? } \\
\begin{array}{lll}\text { ( ) Miopia } & \text { ( ) Catarata } & (\text { ) Glaucoma } \\
\text { ( ) Estrabismo } & (\text { ) Alterações na retina/“vasos" do olho }\end{array}\end{array}$ \\
\hline 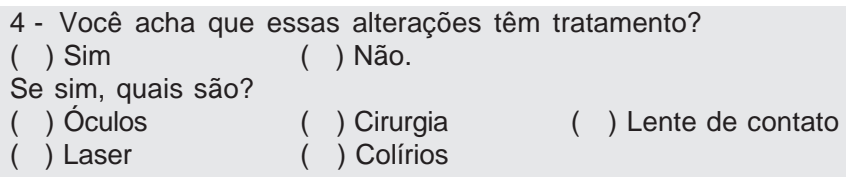 \\
\hline 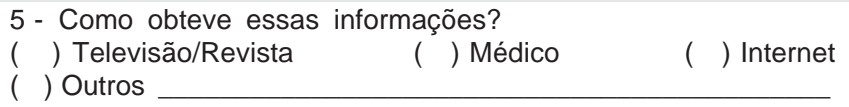 \\
\hline $\begin{array}{l}6 \text { - Qual a frequência que você acha que se deve acompanhar com } \\
\text { o oftalmologista? } \\
\begin{array}{ll}\text { ( ) A cada mês } & (\text { ) } 6 \text { meses a } 1 \text { ano } \\
\text { ( ) Mais de } 1 \text { ano } & \text { ( ) Não há necessidade } \\
\text { Quando foi pela última vez? } & \end{array}\end{array}$ \\
\hline $\begin{array}{l}7 \text { - Você acha que o tempo de doença tem influência nas alterações } \\
\text { oftalmológicas? } \\
\begin{array}{ll}\text { ( ) Sim } & (\text { ) Não }\end{array}\end{array}$ \\
\hline $\begin{array}{l}8 \text { - Você já fez algum tipo de tratamento ocular relacionado às } \\
\text { complicações do diabetes? } \\
\begin{array}{ll}\text { ( ) Sim } & \text { ( ) Não } \\
\text { Qual? }\end{array}\end{array}$ \\
\hline
\end{tabular}




\section{RESULTADOS}

Foram avaliados 100 pacientes, sendo 62 mulheres e 38 homens. A média de idade dos entrevistados foi de 58,64 anos $( \pm 15,64$ anos $)$ e $93 \%$ possuíam diabetes mellitus tipo II.

Dezessete entrevistados tinham diagnóstico de diabetes há menos de 1 ano, 27 de 2 a 5 anos, 16 de 6 a 10 anos e 40, sendo apenas 2 deles pertencentes ao tipo I, tinham há mais de 10 anos (Tabela 1). Quando questionados se o diabetes causava alterações oftalmológicas, 19 pacientes responderam de forma negativa e 11 afirmaram que tinham conhecimento, mas não sabiam referir quais eram essas alterações. A maioria dos pacientes que negaram o conhecimento das alterações pertencia ao tipo II e tinha até 5 anos de doença (26\% até 1 ano, $37 \%$ de 2 a 5 anos, $11 \%$ de 6 a 10 anos e $26 \%$ mais de 10 anos).

Os 70 restantes, que conheciam a existência dessas complicações, mencionaram principalmente a alteração retiniana e a catarata secundária (respectivamente $30 \%$ e $27 \%$ ), acompanhadas pelo glaucoma (20\%), miopia (14\%), e estrabismo $(9 \%)$ (Gráfico 1). Essa ordem de escolha só se modificou no grupo com doença há menos de 1 ano, no qual se evidenciou o glaucoma (25\%) mais prevalente que a catarata (20\%).

Em relação às perguntas sobre a possibilidade de tratamento, 30 pacientes relataram não haver, 7 não sabiam opinar sobre o assunto e 63 responderam que as alterações oculares podiam ser tratadas. Sobre as opções de terapêutica possíveis, a cirurgia foi a mais citada (33\%), em seguida foram o uso de óculos (27\%), aplicação de laser (19\%), uso de colírio (18\%) e lente de contato (3\%) (Gráfico 2). Essa ordem também se manteve entre os diferentes grupos.

A maioria dos pacientes (56\%) associou a aquisição dessas informações à orientação médica, $31 \%$ dos pacientes as

Tabela 1. Tempo de doença dos pacientes entrevistados

\begin{tabular}{lc}
$\begin{array}{l}\text { Tempo de } \\
\text { diabetes mellitus (anos) }\end{array}$ & $\begin{array}{c}\text { Pacientes } \\
\text { entrevistados }\end{array}$ \\
$<1$ & 17 \\
2 a 5 & 27 \\
6 a 10 & 16 \\
> 10 & 40 \\
Total & 100 \\
\hline
\end{tabular}

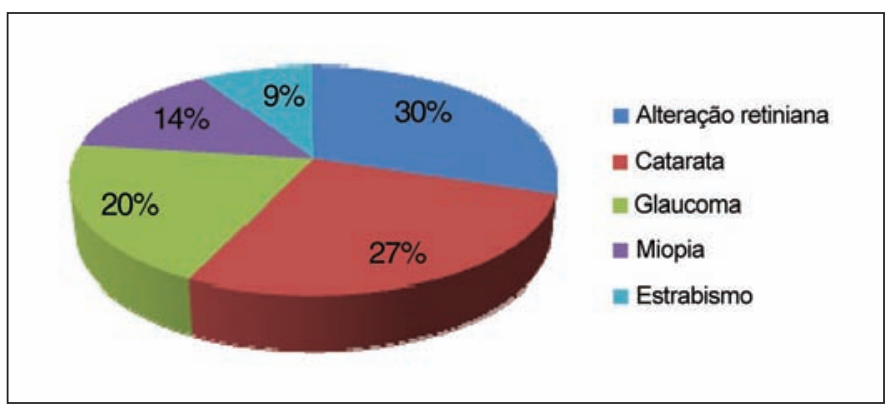

Gráfico 1 - Distribuição das alterações oftalmológicas mencionadas por 70 pacientes obtiveram por outros meios não questionados (como parentes e amigos com diabetes, campanhas etc.) e $13 \%$ citaram a televisão como a fonte de ciência principal.

Cinquenta e seis pacientes entenderam a necessidade do acompanhamento especializado de 6 meses a 1 ano, 7 acharam que o ideal seria o exame mensal e 12 que o intervalo entre os exames poderia ser maior que 1 ano. Apenas 5 pacientes contestaram a obrigatoriedade de acompanhamento oftalmológico periódico.

Embora os pacientes demonstrassem noção sobre a periodicidade ideal do exame, a média do tempo decorrido da ultima consulta foi de cerca de 1 ano e 1 mês ( \pm 1 ano).

Setenta e cinco pacientes alegaram que o tempo de doença apresentou analogia com o aparecimento das complicações oculares, a despeito de 25 que negaram essa relação.

Quando interrogados sobre a realização de tratamentos oftalmológicos prévios, 78 entrevistados negaram e 12 asseguraram já terem necessitado (50\% fotocoagulação a laser, $33 \%$ facectomia, $8 \%$ cirurgia para correção de descolamento de retina e $8 \%$ facectomia e fotocoagulação).

\section{DISCUSS $\tilde{A} O$}

Manter os pacientes diabéticos informados sobre a sua doença é a principal forma de prevenir complicações. No presente estudo, a maior parte dos pacientes que negaram que houvesse alterações oftalmológicas pelo diabetes tinha até cinco anos de doença, o que se mostra preocupante, uma vez que esses pacientes estão, em sua maioria, ainda em situação de prevenção dessas complicações.

Da mesma maneira, daqueles pacientes que responderam ter ciência das complicações oftalmológicas, somente $30 \%$ mencionaram retinopatia diabética e $27 \%$ catarata, que são as principais causas de cegueira e perda na qualidade de vida nesses doentes.

O estudo mostra também equívocos em relação ao tratamento, pois $27 \%$ dos entrevistados que responderam que as complicações eram tratáveis, citaram como opções de terapêutica o uso de óculos, $18 \%$ o uso de colírios e $3 \%$ o de lente de contato. Novamente, os principais tratamentos, como cirurgia e fotocoagulação a laser (respectivamente 33\% e 19\%) mereceriam maior referência.

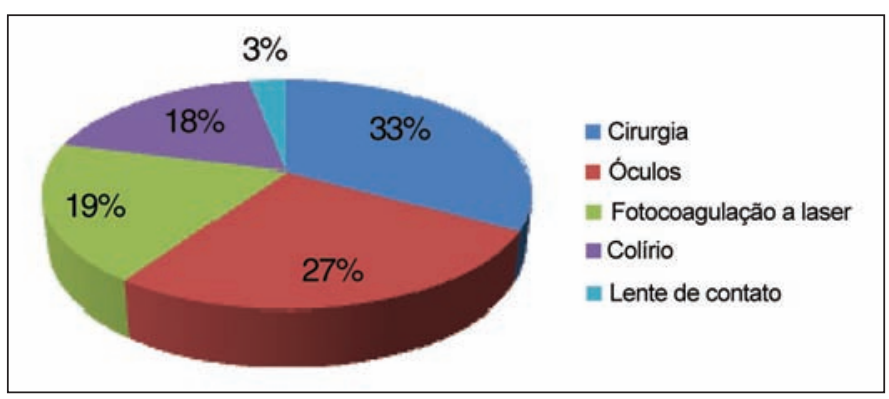

Gráfico 2 - Distribuição dos tratamentos mencionados por 63 pacientes 
Sobre isso, podemos citar a publicação de autores que em 2000, de 5 anos, mascarada, objetivando avaliar se havia relação entre a orientação dos pacientes diabéticos sobre as alterações oculares, através de um "guideline" e a instalação da retinopatia diabética, sob exame de fundoscopia. $\mathrm{Pa}-$ cientes acima de 18 anos, independente do tipo de diabetes, eram questionados se passaram por avaliação oftalmológica no ano anterior e sobre aspectos gerais e cuidados sobre a saúde ocular. Através das respostas, dividiram 2.308 pacientes entre aderentes e não aderentes ao "guideline". Sessenta e nove por cento não apresentaram exame oftalmológico com dilatação no último ano, sendo em sua maioria homens. O perfil dos pacientes não aderentes era os de pouco tempo de doença, tipo II e com aumento do índice de massa corpórea. Noventa e cinco por cento reconheciam que a avaliação oftalmológica anual era necessária (maioria tipo I) e 69\% relataram que foram informados pelo médico quanto a isso $(10 \% \text { por optometristas })^{(8)}$, fato semelhante ao observado no nosso estudo.

Outro artigo, publicado em 1997, recrutou 250 pacientes em tratamento do diabetes tipo I com uso intensivo de insulina e os ofereceu um curso de 23 a $30 \mathrm{~h}$ referente a aspectos dessa doença (controle metabólico, dieta, modo de aplicar insulina, ajuste da dose da insulina para atividades físicas, infecções e complicações). Eles foram acompanhados por um período de 6 meses, compreendendo investigação clínica e psicológica. Comparando-se os parâmetros pré e pós-tratamento, não se observou alterações significantes nos parâmetros clínicos e no uso da insulina, mas se notou um decréscimo na maneira de enfrentar os problemas cotidianos com fatalismo, melhora na relação médico-paciente e maior autonomia no manejo da doença ${ }^{(9)}$.

Diferentes publicações também enfatizam a necessidade de orientação constante dos diabéticos. Em 2008 foi realizado estudo com 86 pacientes de idade média de 23 anos e diagnóstico recente de diabetes tipo I (através de cetoacidose diabética), objetivando a investigação anual durante cerca de 7 anos. Todos receberam, durante a hospitalização, um curso de orientação sobre a doença, seu tratamento e complicações. Os pacientes, além de serem avaliados anualmente, respondiam a um questionário de 20 perguntas sobre qualidade de vida, hábitos e evolução da doença. A avaliação relativa à retinopatia diabética era feita anualmente e classificada de acordo com as normas da Academia Americana de Oftalmologia. Conforme os pontos obtidos no questionário, os estudados eram classificados de A a C (A>16, B entre $12 \mathrm{e}$ 16 e $\mathrm{C}<12$ pontos). Os do grupo A tiveram melhor controle metabólico e menor evolução da microangiopatia. Vinte e cinco por cento dos pacientes ficaram no grupo $\mathrm{C}$, mostrando que não basta um curso de orientação, sendo esta necessária durante todo o acompanhamento do paciente. Entre esses, houve o maior número de pacientes com retinopatia diabética ${ }^{(10)}$.

Pelo fato das complicações oftalmológicas relacionadas ao diabetes, principalmente as retinianas, não serem sintomáticas nas suas formas iniciais, a necessidade de monitoramento, detecção e intervenção precoces são imprescindíveis.

A literatura médica apresenta trabalhos envolvendo essa temática, como o realizado por Ronald Klein, que mostra a necessidade de um "screening" para a retinopatia diabética. Foram selecionados pacientes que seguiram ou não as recomendações do WESDR, um estudo baseado na incidência e prevalência da retinopatia diabética. Divididos em 2 grupos (1- com diagnóstico de DM antes dos 30 anos e em uso de insulina; 2- com diagnóstico após os 30 anos), 1.210 pacientes passaram por exame clínico e oftalmológico e os que apresentavam alterações foram encaminhados ao oftalmologista para acompanhamento. Após 4 anos, repetiu-se os exames em $89 \%$ dos pacientes, com resultados semelhantes ao original. Setenta e seis por cento não tiveram avaliação oftalmológica desde a primeira análise. Daqueles recomendados a procurar acompanhamento especializado, $77 \%$ o fizeram. Presume-se, dessa forma, que a orientação dos pacientes quanto à necessidade de avaliação oftalmológica é importante no diagnóstico precoce das lesões oculares ${ }^{(11)}$.

Uma vez já estabelecidas as lesões, o acompanhamento do paciente deve ser ainda mais próximo e deve ser disponibilizado a ele as melhores opções de tratamento. Em 2002, foi feita uma revisão bibliográfica de trabalhos mostrando que a intervenção precoce pela fotocoagulação prevenia a perda visual em pacientes com retinopatia diabética e, mesmo assim, um número grande de pacientes permanecia sem diagnóstico e consequentemente sem a terapêutica ideal. Os estudos WESDR e DCCT mostraram redução do risco de desenvolvimento de retinopatia diabética $(\mathrm{RD})$ em pacientes com tratamento clínico intensivo precoce. O UKPDS também mostrou diminuição de $12 \%$ na progressão da RD e na necessidade de fotocoagulação a laser. Há também relação da pressão arterial como fator preditor na progressão da RD e incidência do tipo proliferativo e da elevação dos triglicerídeos com a formação de exsudatos duros ${ }^{(12)}$.

Na nossa avaliação, o fato da maioria dos pacientes (56\%) relatarem que obtiveram as informações sobre as complicações oculares da doença por orientação médica mostra que formas de melhorar a relação médico-paciente são importantes. Uma alternativa avaliada por alguns autores em 2001 foi a introdução de um serviço informatizado ("telecare") para o acompanhamento de pacientes diabéticos, em esquema de tratamento intensivo com insulina. Assim, o estudo investiga melhor controle da doença e aderência ao tratamento pela forma informatizada ou convencional. Concluiu-se que o "telecare" intensificou a relação médico-paciente, proporcionando maior liberdade no manejo da doença e segurança pelo fato dos envolvidos poderem recorrer ao auxílio especializado com mais facilidade. Em relação aos parâmetros metabólicos, não houve diferença estatística significativa entre os grupos, sugerindo que essa se deve à intensificação no cuidado à doença em ambos os grupos ${ }^{(13)}$.

No presente estudo, pelo baixo nível socioeconômico da população avaliada, método como relatado acima pode pare- 
cer impraticável, mas é bem visto como idéia de aprimoramento do tão importante contato do médico com seu paciente.

\section{CONCLUSÃO}

Pelos dados obtidos no nosso trabalho, podemos concluir que não é suficiente apenas a orientação dos pacientes da necessidade do exame oftalmológico, pois muitos desses pacientes não sabiam referir as principais complicações e tratamentos decorrentes do diabetes.

Dessa forma, pode-se concluir que falta informação especializada a esses pacientes e orientação da necessidade do tratamento precoce para a prevenção da cegueira.

\section{ABSTRACT}

Purpose: To evaluate the knowledge of diabetic patients about ocular changes that could be caused by diabetes mellitus. Methods: A hundred diabetic patients were interviewed in the period of three weeks. Those patients had a scheduled appointment at Endocrinology Sector of Guilherme Álvaro Hospital. The patients answered eight questions about ocular changes that could be caused by diabetes mellitus. Results: Sixty-two female and 38 male, $93 \%$ with type II diabetes and mean age of 58.64 years ( \pm 15.64 years) were evaluated. Forty patients had the diabetes diagnosed for more than 10 years. Seventy per cent of the interviewed patients presented some knowledge about diabetic ocular changes. The questions about treatment demonstrated that 30 patients did not know any kind of treatment and 63 assured that diabetic ocular changes could be treated. Regarding the treatment options, surgery was mentioned by $33 \%$ of the patients while, $27 \%$ mentioned glasses, $19 \%$ laser therapy and $18 \%$ mentioned eye drops. The majority (56\%) associated their knowledge to doctor's orientation. Some of them understood that they should be examined every 6 months because they learned that ocular changes are likely to occur as the time of diabetes diagnosis increase. Seventy-eight patients denied any ocular treatment and 12 had already performed some treatment. Conclusion: This study demonstrated that $81 \%$ of the diabetic patients presents some knowledge about diabetes ocular changes, but they do not have precise informations.

Keywords: Diabetes mellitus/complications; Diabetic retinopathy; Cataract/epidemiology; Health knowledge, attitudes, practice

\section{REFERÊNCIAS}

1. Goldman L, Bennett JC. Tratado de Medicina Interna Cecil. Rio de Janeiro: Guanabara Koogan; 2001.

2. Sociedade Brasileira de Diabetes. Manual da abordagem nutricional em Diabetes. Plano de reorganização da atenção à hipertensão arterial e ao Diabetes Mellitus. [Internet]. [citado 2000 Maio 24]. Disponível em: http://dtr2004. saude.gov.br/dab/hipertensaodiabetes/ publicacoes.php

3. Rede Interagencial de Informações pela Saúde - RIPSA. Indicadores de morbidade e fatores de risco. D.10 Taxa de prevalência de diabetes mellitus [Internet]. Brasília: Ministério da Saúde. [citado 2207 Nov 24]. Disponível em: http://tabnet. datasus.gov.br/cgi/idb2006/d10.htm

4. Conselho Brasileiro de Oftalmologia. Retinopatia diabética [Internet]. São Paulo: CBO. [citado 2008 Dez 18]. Disponível em: http://www.cbo.com.br/pacientes/ doencas/doencas_retinopatia_diabetica.htm

5. Ministério da Saúde. Secretaria de Políticas da Saúde. Plano de reeorganização da atenção à hipertensão arterial e ao diabetes mellitus. Programa de educação permanente em hipertensão arterial e diabetes mellitus para os municípios com população acima de 100 mil habitantes [Internet]. Brasília; 2002; [citado 2008 Npv 20] Disponível em: http://dtr2004.saude.gov.br/dab/docs/publicacoes/geral/ projeto_educacao_permanente-maio14.pdf

6. Preti RC, Saraiva F, Trein Junior JA, Takahashi WY, Silva ME. How much information do medical practitioners and endocrinologists have about diabetic retinopathy? Clinics. (São Paulo). 2007;62(3):273-8.

7. Kanski JJ. Oftalmologia Clínica. Rio de Janeiro: Elsevier; 2004.

8. Schoenfeld ER, Greene JM, Wu SY, Leske MC. Patterns of adherence to diabetes vision care guidelines: baseline findings from the Diabetic Retinopathy Awareness Program. Ophthalmology. 2001;108(3):563-71.

9. Langewitz W, Wössmer B, Iseli J, Berger W. Psychological and metabolic improvement after an outpatient teaching program for functional intensified insulin therapy (FIT). Diabetes Res Clin Pract. 1997;37(3):157-64.

10. Araszkiewicz A, Zozulinska-Ziolkiewicz D, Trepinska M, Wierusz-Wysocka B. Knowledge after five-day teaching program in intensive insulin therapy performed at the onset of type 1 diabetes influence the development of late diabetic complications. Diabetes Res Clin Pract. 2008;81(1):61-7.

11. Newcomb PA, Klein R. Factors associated following diabetic with compliance eye screening. J Diabetic Complications. 1990;4(10):8-14.

12. Klein R. Prevention of visual loss from diabetic retinopathy. Surv Ophthalmol. 2002;47(Suppl 2):S246-52.

13. Biermann E, Dietrich W, Rihl J, Standl E. Are there time and cost savings by using telemanagement for patients on intensified insulin therapy? A randomised, controlled trial. Computer Methods and Programs Biomed. 69(2):137-46. 